\title{
Upregulation of SLC2A3 gene and prognosis in colorectal carcinoma: analysis of TCGA data
}

Eunyoung Kim ${ }^{1 \dagger}$, Sohee Jung ${ }^{2 \dagger}$, Won Seo Park ${ }^{3}$, Joon-Hyop Lee ${ }^{4}$, Rumi Shin ${ }^{5}$, Seung Chul Heo ${ }^{5}$, Eun Kyung Choe ${ }^{6}$, Jae Hyun Lee ${ }^{7}$, Kwangsoo Kim ${ }^{2 *}$ and Young Jun Chai ${ }^{5^{*}}$

\begin{abstract}
Background: Upregulation of SLC2A genes that encode glucose transporter (GLUT) protein is associated with poor prognosis in many cancers. In colorectal cancer, studies reporting the association between overexpression of GLUT and poor clinical outcomes were flawed by small sample sizes or subjective interpretation of immunohistochemical staining. Here, we analyzed mRNA expressions in all 14 SLC2A genes and evaluated the association with prognosis in colorectal cancer using data from the Cancer Genome Atlas (TCGA) database.
\end{abstract}

Methods: In the present study, we analyzed the expression of SLC2A genes in colorectal cancer and their association with prognosis using data obtained from the TCGA for the discovery sample, and a dataset from the Gene Expression Omnibus for the validation sample.

Results: SLC2A3 was significantly associated with overall survival (OS) and disease-free survival (DFS) in both the discovery sample (345 patients) and validation sample (501 patients). High SLC2A3 expression resulted in shorter OS and DFS. In multivariate analyses, high SLC2A3 levels predicted unfavorable OS (adjusted HR 1.95, 95\% Cl 1.22-3.11; $P=0.005$ ) and were associated with poor DFS (adjusted HR 1.85, 95\% Cl 1.10-3.12; $P=0.02$ ). Similar results were found in the discovery set.

Conclusion: Upregulation of the SLC2A3 genes is associated with decreased OS and DFS in colorectal cancer patients. Therefore, assessment of SLC2A3 gene expression may useful for predicting prognosis in these patients.

Keywords: Colorectal cancer, Solute carrier 2A (SLC2A), Glucose transporter (GLUT), The cancer genome atlas (TCGA), Prognosis

\section{Background}

Colorectal cancer (CRC) is the third most common cancer and the fourth-leading cause of cancer death in the world $[1,2]$. Most CRCs originate from non-cancerous lesions by one or a combination of three different mechanisms: chromosomal instability, $\mathrm{CpG}$ island methylator phenotype, and microsatellite instability [3]. Biomarkers of these cytogenetic alterations are of interest for diagnosis,

\footnotetext{
* Correspondence: kksoo@snuh.org; kevinjoon@naver.com

${ }^{\dagger}$ Eunyoung Kim and Sohee Jung have contributed equally as co-first authors ${ }^{2}$ Division of Clinical Bioinformatics, Biomedical Research Institute, Seoul National University Hospital, Seoul, Republic of Korea

${ }^{5}$ Department of Surgery, Seoul Metropolitan Government - Seoul National University Boramae Medical Center, 20 Boramae-ro 5-gil, Dongjak-gu, Seoul 156-70, Republic of Korea

Full list of author information is available at the end of the article
}

prognostication, and anticancer drug development targeting CRC [4]. Despite research efforts, genetic biomarkers currently have limited value as diagnostic or prognostic markers [5].

Among the biomarkers, the solute carrier 2A (SLC2A) gene family that encodes glucose transporter (GLUT) proteins has been widely investigated. GLUT proteins facilitate glucose influx into cancer cells which is necessary for cancer cell proliferation. Upregulation of $S L C 2 A$ genes is associated with poor prognosis in many cancers, including hepatocellular carcinoma, non-small cell lung cancer, and thyroid carcinoma [6-8].

An association between overexpression of the subtypes of GLUT proteins and poor clinical outcomes has been reported in CRC [9]. However, these studies were flawed

(c) The Author(s). 2019 Open Access This article is distributed under the terms of the Creative Commons Attribution 4.0 International License (http://creativecommons.org/licenses/by/4.0/), which permits unrestricted use, distribution, and reproduction in any medium, provided you give appropriate credit to the original author(s) and the source, provide a link to the Creative Commons license, and indicate if changes were made. The Creative Commons Public Domain Dedication waiver (http://creativecommons.org/publicdomain/zero/1.0/) applies to the data made available in this article, unless otherwise stated. 
by small sample sizes or subjective interpretation of immunohistochemical (IHC) staining. In this study, we analyzed the mRNA expression of all $14 S L C 2 A$ genes (corresponding to 14 GLUT proteins) and evaluated the associations with prognosis in CRC using data from the Cancer Genome Atlas (TCGA) database.

\section{Methods}

\section{Data acquisition}

The TCGA CRC data was downloaded from cBioPortal for Cancer Genomics (http://www.cbioportal.org/). The dataset contains survival data with clinical information, somatic mutations, and mRNA expression counts. For validation, we obtained independent microarray datasets (GSE39582) from the Gene Expression Omnibus (GEO). mRNA counts of the validation set were measured by the Affymetrix Human Genome U133 Plus 2.0 Array in a $\log 2$ scale. Gene expression of the discovery set was measured by Illumina HiSeq platform and transformed into $\log 2$ scale. According to the publication guidelines, the datasets may be used for publication without restriction or limitation (https://cancergenome.nih.gov/publications/publicationguidelines, https://www.ncbi.nlm.nih. gov/geo/info/disclaimer.html).

\section{Statistical analysis}

The putative associations between conventional clinical pathology parameters (age at diagnosis, sex, American Joint Committee on Cancer (AJCC) 7th edition TNM stage, microsatellite instability, and mutational status of KRAS (v-Ki-ras2 Kirsten rat sarcoma viral oncogene homolog) and BRAF (v-Raf murine sarcoma viral oncogene homolog B1 genes) and survival outcome were assessed by Chi-square tests. The Cox proportional-hazards model was used to identify genes associated with survival and to estimate mortality hazard ratios (HRs). The optimal cut-off points for $S L C 2 A 3$ expression used to divide patients into low-risk and high-risk groups were determined using the MaxStat package of $\mathrm{R}$ software (Maximally selected Rank Statistics). Maxstat computes the maximally selected log-rank statistic to identify the cutpoint which provides the best separation (in which the standardized statistics take their maximum) into two groups. Kaplan-Meier analysis was performed to estimate the survival curves of the different subgroups and the log-rank test (Mantel-Cox) was used to compare the curve. Statistical analyses were performed using R statistical software (version 3.4.1) [10]. All $P$-value were two sided. A P-value less than 0.05 was considered statistically significant.

\section{Results}

\section{Patient demographics}

Our study sample comprised 846 patients. Patient characteristics of the discovery and validation set are shown in Table 1 .
Table 1 Patient demographics

\begin{tabular}{|c|c|c|}
\hline & $\begin{array}{l}\text { Discovery Set } \\
(\text { TCGA, N=345) }\end{array}$ & $\begin{array}{l}\text { Validation Set } \\
(\text { GSE39582, N=501) }\end{array}$ \\
\hline \multicolumn{3}{|l|}{ Age, yr } \\
\hline Median & 67 & 68.1 \\
\hline Interquartile range & $(55-75)$ & $(59-76)$ \\
\hline \multicolumn{3}{|l|}{ Sex } \\
\hline Female & 157 (46\%) & $229(46 \%)$ \\
\hline Male & $188(54 \%)$ & $272(54 \%)$ \\
\hline \multicolumn{3}{|l|}{ AJCC TNM Stage } \\
\hline । & $54(16 \%)$ & $31(6 \%)$ \\
\hline$\|$ & 130 (38\%) & 244 (49\%) \\
\hline III & $111(32 \%)$ & 166 (33\%) \\
\hline IV & 50 (14\%) & $60(12 \%)$ \\
\hline \multicolumn{3}{|l|}{ Microsatellite instability } \\
\hline MSI-L and MSS & 291 (84\%) & \\
\hline MSI-H & $54(16 \%)$ & \\
\hline \multicolumn{3}{|l|}{ BRAF status } \\
\hline V600E & $50(14 \%)$ & $49(10 \%)$ \\
\hline Wild-type & 295 (86\%) & $452(90 \%)$ \\
\hline \multicolumn{3}{|l|}{ KRAS status } \\
\hline Mutant & $144(42 \%)$ & 198 (40\%) \\
\hline Wild-type & $201(58 \%)$ & $303(60 \%)$ \\
\hline \multicolumn{3}{|l|}{ OS event } \\
\hline Event & $78(23 \%)$ & 171 (34\%) \\
\hline Non-event & $267(77 \%)$ & $330(66 \%)$ \\
\hline \multicolumn{3}{|l|}{ OS months } \\
\hline Median & 31.4 & 56.5 \\
\hline Range & $(0-147.9)$ & $(0-201)$ \\
\hline \multicolumn{3}{|l|}{ DFS event } \\
\hline Event & $83(24 \%)$ & $150(30 \%)$ \\
\hline Non-event & $222(64 \%)$ & 346 (69\%) \\
\hline Not available & $40(12 \%)$ & $5(1 \%)$ \\
\hline \multicolumn{3}{|l|}{ DFS months } \\
\hline Median & 21 & 44 \\
\hline Range & (0-148) & $(0-201)$ \\
\hline
\end{tabular}

The TCGA sample of 345 patients were the discovery set, and the GSE39582 sample of 501 patients were the validation set.

\section{Association between clinical parameters and survival outcome}

The associations between clinical variables and OS and DFS in the discovery set are summarized in Table 2. Age $>65$ was associated with worse OS compared to age $\leq 65 \quad(P<0.001)$. TNM stage III and IV was 
Table 2 Association between the clinicopathological characteristics and survival outcome

\begin{tabular}{|c|c|c|c|c|c|c|}
\hline & \multicolumn{3}{|c|}{ Overall survival } & \multicolumn{3}{|c|}{ Disease-free survival } \\
\hline & Non-event & Event & $P$ value $^{a}$ & Non-event & Event & $P$ value $^{a}$ \\
\hline \multicolumn{7}{|l|}{ Age } \\
\hline$<=65$ & 141 & 23 & $<0.001$ & 115 & 43 & 1 \\
\hline$>65$ & 126 & 55 & & 107 & 40 & \\
\hline \multicolumn{7}{|l|}{ Sex } \\
\hline Female & 124 & 33 & 0.606 & 103 & 34 & 0.472 \\
\hline Male & 143 & 45 & & 119 & 49 & \\
\hline \multicolumn{7}{|l|}{ AJCC TNM stage } \\
\hline | and || & 157 & 27 & $<0.001$ & 137 & 32 & $<0.001$ \\
\hline III and IV & 110 & 51 & & 85 & 51 & \\
\hline \multicolumn{7}{|l|}{ MSI } \\
\hline MSI-L and MSS & 224 & 67 & 0.802 & 184 & 72 & 0.520 \\
\hline MSI-H & 43 & 11 & & 38 & 11 & \\
\hline \multicolumn{7}{|l|}{ BRAF status } \\
\hline Wild-type & 230 & 65 & 0.662 & 188 & 74 & 0.416 \\
\hline Mutant & 37 & 13 & & 34 & 9 & \\
\hline \multicolumn{7}{|l|}{ KRAS status } \\
\hline Wild-type & 154 & 47 & 0.783 & 133 & 43 & 0.252 \\
\hline Mutant & 113 & 31 & & 89 & 40 & \\
\hline
\end{tabular}

${ }^{a}$ Calculated using the Chi-square test

associated with OS $(\mathrm{P}<0.001)$ and DFS $(\mathrm{P}<0.001)$. Other clinicopathological factors (sex, MSI, $B R A F$, or $K R A S$ status) were not associated with OS or DFS.

\section{Prognostic value of SLC2A3}

Table 3 displays the associations between the mRNA expression values of SLC2 family genes and survival outcomes. SLC2A3 was significantly associated with both OS $(P=0.013)$ and DFS $(P=0.014)$. There were associations between the expression of SLC2A6 and SLC2A7 with worse $\mathrm{OS}(P=0.048$ and 0.019$)$; and $S L C 2 A 1$ with worse DFS $(P=0.018)$.

Patients were categorized into high and low SLC2A3 expression groups according to the cut-off value determined by Maxstat method. The high expression group had worse OS $(P=0.005)$ and DFS $(P=0.002)$ compared to the low expression group (Fig. 1).

We explored the association between $S L C 2 A 3$ expression and survival outcome in a multivariate context (Table 4). In multivariate Cox regression analysis, after adjusting for clinical factors that were significantly associated with survival outcome (OS: age > 65, AJCC stage III and IV, DFS; AJCC stage III and IV), SLC $2 A 3$ remained an independent poor prognostic factor for OS and DFS. SLC2A3 level is associated with shorter OS with an adjusted hazard ratio of 1.98 (95\% CI: 1.24-3.17; $P=0.004$ ) and with poor disease-free survival (adjusted HR: 1.85 , 95\% CI: $1.10-3.12 ; P=0.020)$.
Table 3 Univariate cox regression analysis of SLC2 family genes for OS and DFS

\begin{tabular}{|c|c|c|c|c|}
\hline \multirow[t]{2}{*}{ Gene $^{a}$} & \multicolumn{2}{|l|}{ OS } & \multicolumn{2}{|l|}{ DFS } \\
\hline & $\mathrm{HR}(95 \% \mathrm{Cl})$ & $P$ value ${ }^{b}$ & HR (95\% Cl) & $P$ value ${ }^{b}$ \\
\hline SLC2A1 & $1.13(0.91-1.39)$ & 0.271 & $1.28(1.04-1.57)$ & 0.018 \\
\hline$S L C 2 A 2$ & $0.82(0.61-1.09)$ & 0.169 & $0.99(0.8-1.24)$ & 0.957 \\
\hline$S L C 2 A 3$ & $1.33(1.06-1.66)$ & 0.013 & $1.32(1.06-1.64)$ & 0.014 \\
\hline SLC2A4 & $1.12(0.9-1.39)$ & 0.302 & $1.02(0.82-1.27)$ & 0.864 \\
\hline SLC2A5 & $1.17(0.94-1.47)$ & 0.159 & $1.04(0.84-1.29)$ & 0.703 \\
\hline SLC2A6 & $1.24(1-1.54)$ & 0.048 & $1.07(0.87-1.32)$ & 0.503 \\
\hline$S L C 2 A 7$ & $1.23(1.03-1.46)$ & 0.019 & $1.06(0.87-1.29)$ & 0.562 \\
\hline$S L C 2 A 8$ & $0.82(0.67-1.01)$ & 0.066 & $0.95(0.78-1.17)$ & 0.646 \\
\hline SLC2A9 & $0.86(0.7-1.05)$ & 0.135 & $1.00(0.82-1.23)$ & 0.965 \\
\hline SLC2A10 & $0.94(0.76-1.17)$ & 0.597 & $1.02(0.82-1.27)$ & 0.876 \\
\hline SLC2A11 & $1.14(0.9-1.43)$ & 0.277 & $1.11(0.88-1.4)$ & 0.369 \\
\hline SLC2A12 & $1.05(0.84-1.32)$ & 0.643 & $1.06(0.86-1.32)$ & 0.574 \\
\hline SLC2A13 & $0.89(0.71-1.11)$ & 0.305 & $1.02(0.82-1.27)$ & 0.835 \\
\hline SLC2A14 & $1.19(0.96-1.49)$ & 0.115 & $1.18(0.96-1.46)$ & 0.123 \\
\hline
\end{tabular}

Abbreviations: $\mathrm{HR}$, hazard ratio; $\mathrm{Cl}$, confidence interval

anit of measure is $\log 2$ of gene expression intensity

${ }^{\mathrm{b}}$ Calculated using the Wald test 
(a)

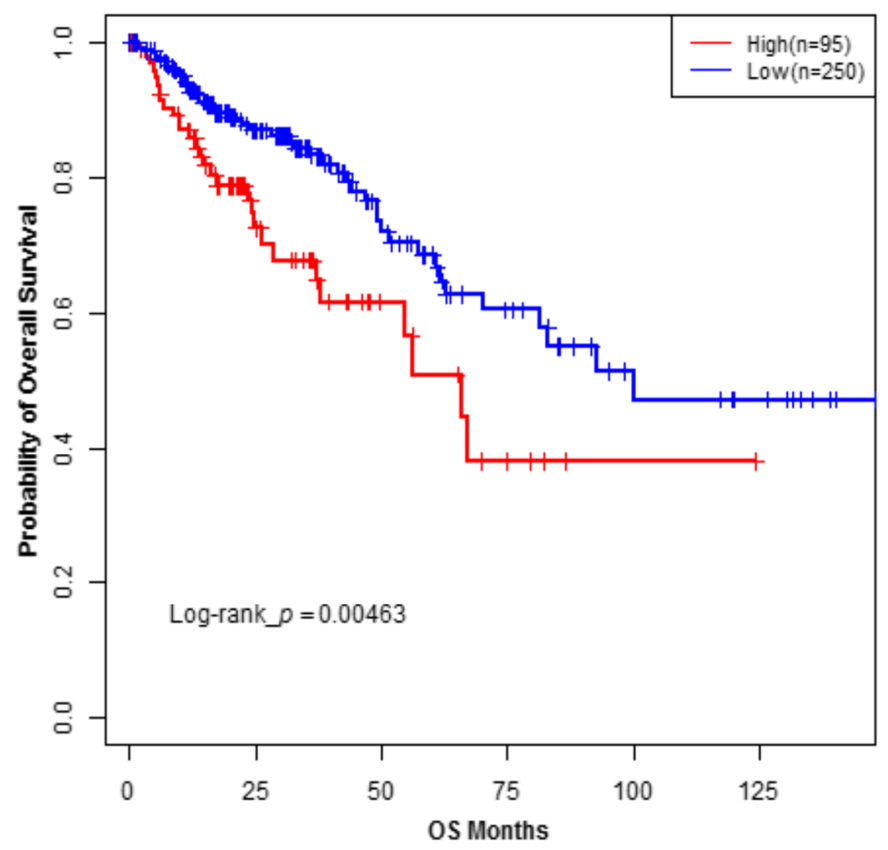

(b)

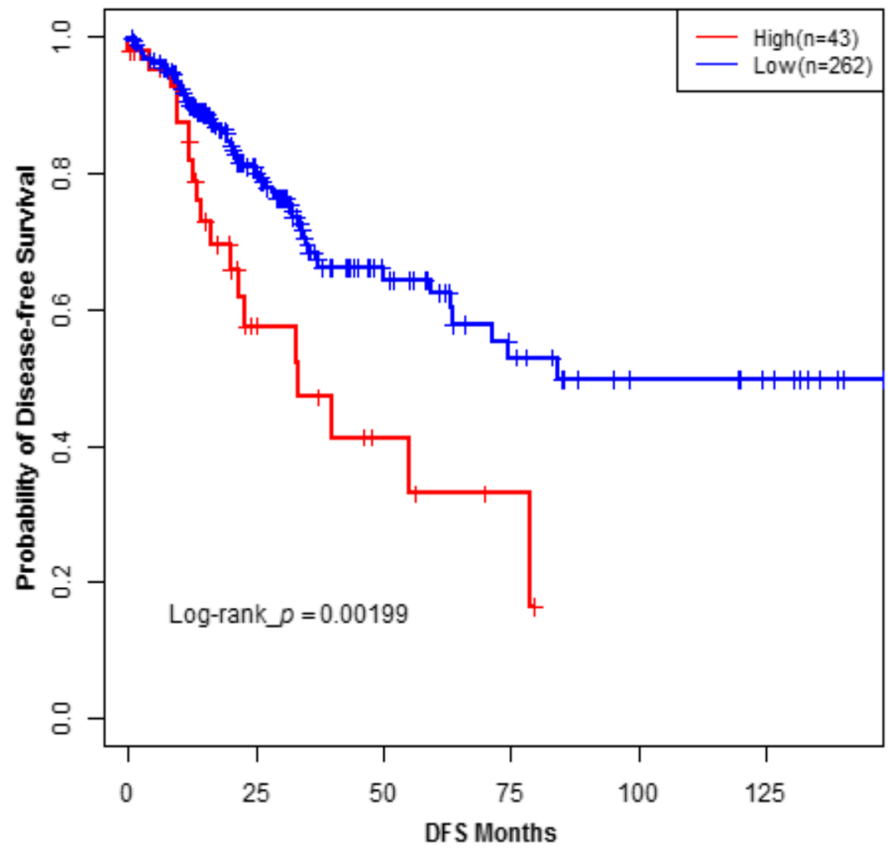

Fig. 1 Kaplan-Meier survival analysis of colorectal cancer patients stratified by SLC2A3 expression levels (Discovery set). Overall survival (a) and disease-free survival (b) curve of patients in discovery set with high versus low SLC2A3 expression levels. P-values for significance of difference between high and low expression were calculated using the log-rank test

\section{Validation set analysis}

$S L C 2 A 3$ was significantly associated with both OS $(P=0.005)$ and DFS $(P=0.024)$. There was associations between the expression of $S L C 2 A 1$ with worse DFS $(P=0.015)$, but SLC2A6 was not associated with worse OS $(P=0.940)$. The expression of $S L C 2 A 7$ was not provided. Patients in validation set were categorized into high vs. low SLC2A3 expression according to the cut-off point. High expression led 
Table 4 Univariate and multivariate Cox regression analyses of SLC2A3 expression and the clinicopathological factors in the discovery set (TCGA COADREAD cohort)

\begin{tabular}{|c|c|c|c|c|}
\hline & \multicolumn{2}{|l|}{ Overall survival } & \multicolumn{2}{|l|}{ Disease-free survival } \\
\hline & $\mathrm{HR}(95 \% \mathrm{Cl}$ of $\mathrm{HR})$ & $P$ value $^{a}$ & $\mathrm{HR}(95 \% \mathrm{Cl}$ of $\mathrm{HR})$ & $P$ value \\
\hline SLC2A3 low & 1.00 (Reference) & & 1.00 (Reference) & \\
\hline UVA SLC2A3 high & $1.93(1.21-3.07)$ & 0.005 & $2.20(1.32-3.68)$ & 0.003 \\
\hline MVA SLC2A3 high & $1.98(1.24-3.17)$ & 0.004 & $1.85(1.10-3.12)$ & 0.020 \\
\hline Age $<=65$ & 1.00 (Reference) & & & \\
\hline Age $>65$ & $2.61(1.59-4.28)$ & $<0.001$ & & \\
\hline AJCC stage I, II & 1.00 (Reference) & & 1.00 (Reference) & \\
\hline AJCC stage III, IV & $3.17(1.96-5.11)$ & $<0.001$ & $2.48(1.58-3.89)$ & $<0.001$ \\
\hline
\end{tabular}

Abbreviations: $H R$ hazard ratio, $\mathrm{Cl}$ confidence interval, UVA univariate, MVA multivariate

${ }^{\text {a }}$ Calculated using the Wald test

to worse OS $(P=0.003)$ and DFS $(P=0.021)$ (Fig. 2$)$. In the multivariate Cox regression analysis, $S L C 2 A 3$ expression is associated with shorter OS (adjusted HR 1.50, 95\% CI: 1.112.03; $P=0.009$ ) and DFS (adjusted HR 1.38, 95\% CI: 1.00 1.91; $P=0.048$ ) after adjusting for clinical factors (age, AJCC stage for OS, AJCC stage for DFS) (Table 5).

\section{Prognostic value of SLC2A1}

Patients were categorized into high and low SLC2A1 expression groups according to the cut-off value determined by Maxstat method. The high expression group had worse DFS in both Discovery set and Validation set ( $P=0.001$ and $<0.001$, respectively) compared to the low expression group (Fig. 3). We explored the association between $S L C 2 A 1$ expression and DFS in a multivariate context of Discovery set and Validation set. In multivariate Cox regression analysis, after adjusting for AJCC TNM stage III and IV that were significantly associated with DFS, SLC2A1 remained an independent poor prognostic factor for DFS in both Discovery set (adjusted HR 1.83, 95\% CI: $1.12-3.01 ; P=0.017)$ and Validation set (adjusted HR 1.65, 95\% CI: 1.14-2.40; $\mathrm{P}=0.009$ ).

\section{Discussion}

Most cancer cells favor glycolytic energy metabolism over mitochondrial metabolism and oxidative phosphorylation chain for energy production, even in the presence of oxygen (Warburg effect). This explains why malignant cells overexpress GLUT family proteins, which is a plasma membrane transport system. Glucose can be translocated into the cell only via GLUT proteins. Expression and subcellular distribution of GLUT proteins are regulated by different signaling molecules and pathways such as PI3K, HIF, p53, Myc, and AMPK LBk1 [11, 12].

In the current study, we used the TCGA database to explore the clinical significance of SLC2 family genes in CRC. IHC staining of GLUT expression in cancer cells can be diverse which is a downside with IHC staining. This may be caused by incorrectly interpreted IHC stain results or different GLUT positive thresholds [9]. Messenger RNA-gene expression analysis from TCGA data is superior to IHC and may best predict cancer prognosis in TCGA data [13]. The current study is the first to report the expression of $S L C 2 A$ genes in CRC using the TCGA database.

In the current study, age and AJCC TNM stage were associated with survival in CRC patient. As expected, in this study, age $>65$ was linked with worse prognosis for CRC patients. This may be due to aging itself, or due to co-morbidities such as cardiovascular disease or postoperative complications [14]. Older patients are less likely to be treated with resectional surgery than younger patients and have poorer survival outcomes [15]. We included age $>65$ as a factor for multivariate analysis because age $>65$ is useful for prognostication. We found that AJCC stage III and IV, which has nodal metastasis or organ metastasis, was associated with OS and DFS in the TCGA database. AJCC TNM stage was established based on OS, and lymph node involvement in itself is considered to have a strong influence on OS and DFS in CRC [16]. In oral squamous cell carcinoma and papillary thyroid carcinoma, GLUT3 was known to be a prognostic marker for OS, and was associated with advanced cancer stage (AJCC TNM stage III and IV) which has nodal metastasis $[8,17]$. In current study, we found high expression of $S L C 2 A 3$ was a prognostic factor for predicting OS and DFS in CRC and was not associated with AJCC TNM stage. Similar to current findings, GLUT3 is a significant marker of poor prognosis in laryngeal carcinoma, with no significant differences in nodal or distant metastasis between the GLUT3 negative and GLUT3 positive groups [18]. Since $S L C 2 A 3$ or GLUT3 genes are associated with prognosis in CRC as well as thyroid or laryngeal carcinoma, it would be worthwhile to investigate whether these cancers have similar GLUT-dependent metabolic pathways.

In the current study, we analyzed the mRNA expression values of all $14 S L C 2 A$ family genes and found that 
(a)

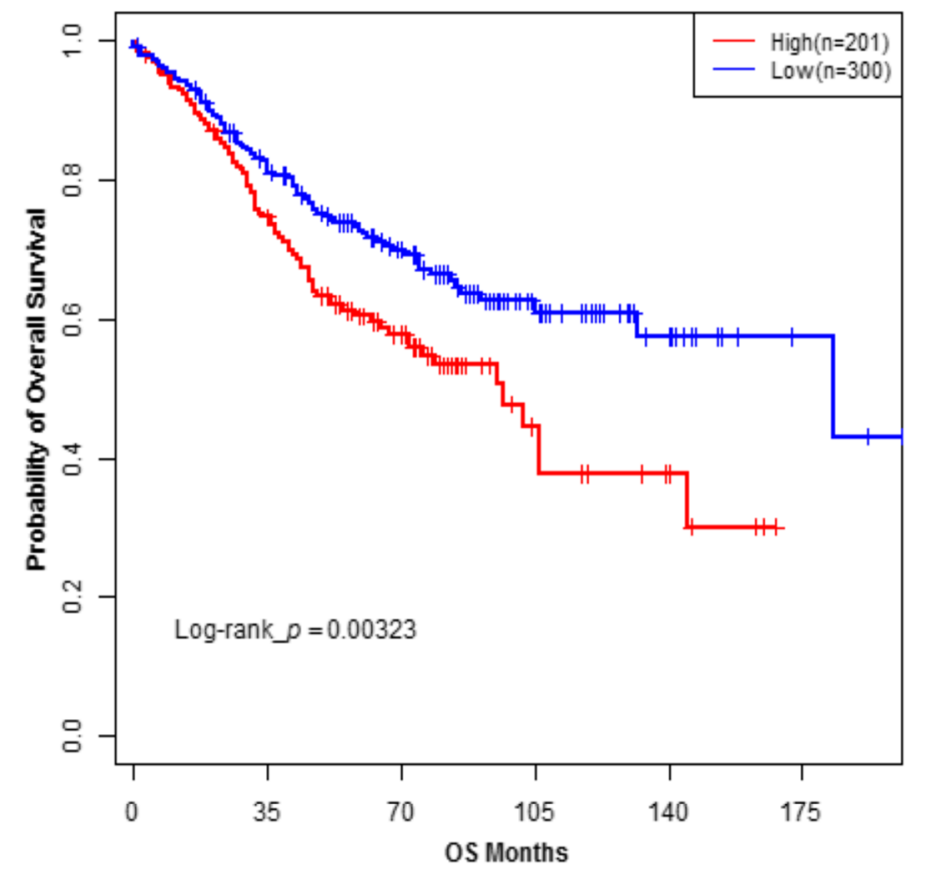

(b)

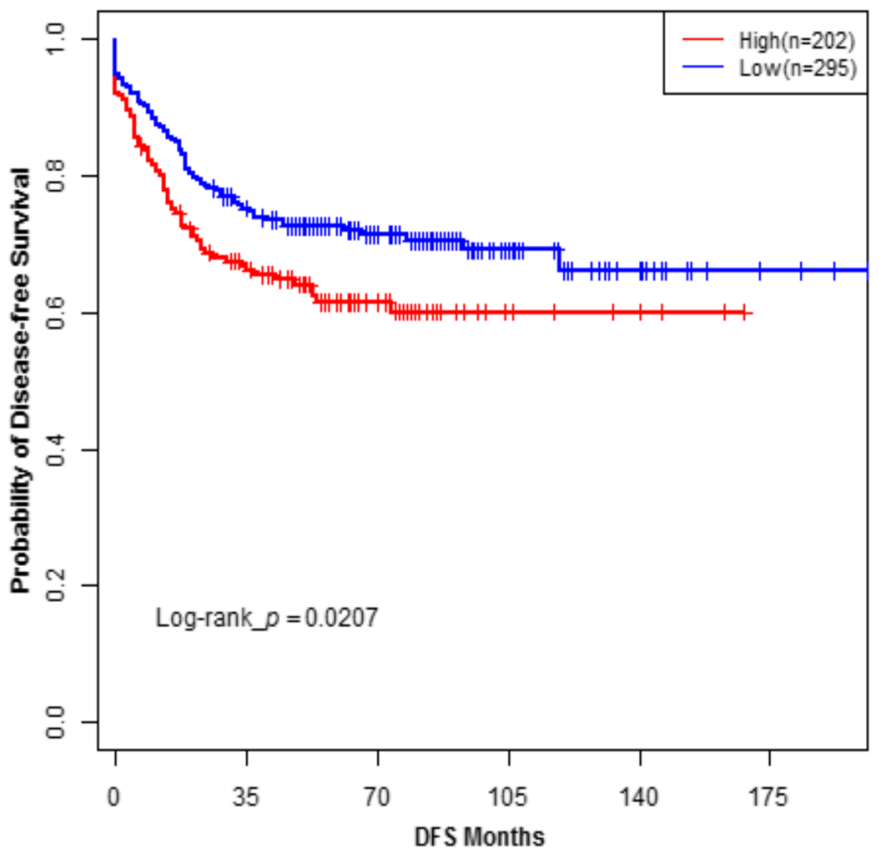

Fig. 2 Kaplan-Meier survival analysis of colorectal cancer patients stratified by SLC2A3 expression levels (Validation set). Overall survival (a) and disease-free survival (b) curve of patients in validation set with high versus low SLC2A3 expression levels. P-values for significance of difference between high and low expression were calculated using the log-rank test

$S L C 2 A 3$ is independently associated with both OS and DFS after adjusting for age and AJCC TNM stage in CRC patients. Among the GLUT family, GLUT1 is associated with prognosis in several cancers, including CRC. GLUT3 has high affinity glucose uptake, similar to GLUT1 [19]. GLUT3 is overexpressed in human 
Table 5 Univariate and multivariate Cox regression analyses of SLC2A3 expression and the clinicopathological factors in the validation set (GSE39582)

\begin{tabular}{|c|c|c|c|c|}
\hline & \multicolumn{2}{|l|}{ Overall survival } & \multicolumn{2}{|c|}{ Disease-free survival } \\
\hline & $\mathrm{HR}(95 \% \mathrm{Cl}$ of $\mathrm{HR})$ & $P$ value $^{a}$ & $\mathrm{HR}(95 \% \mathrm{Cl}$ of $\mathrm{HR})$ & $P$ value $^{\mathrm{a}}$ \\
\hline SLC2A3 low & 1.00 (Reference) & & 1.00 (Reference) & \\
\hline UVA SLC2A3 high & $1.57(1.16-2.12)$ & 0.004 & $1.46(1.06-2.01)$ & 0.021 \\
\hline MVA SLC2A3 high & $1.50(1.11-2.03)$ & 0.009 & $1.38(1.00-1.91)$ & 0.048 \\
\hline Age $<=65$ & 1.00 (Reference) & & & \\
\hline Age $>65$ & $1.39(1.01-1.91)$ & 0.043 & & \\
\hline AJCC stage I, II & 1.00 (Reference) & & 1.00 (Reference) & \\
\hline AJCC stage III, IV & $1.82(1.34-2.46)$ & $<0.001$ & $2.84(2.03-3.98)$ & $<0.001$ \\
\hline
\end{tabular}

Abbreviations: $H R$ hazard ratio, $\mathrm{Cl}$ confidence interval, UVA univariate; MVA,multivariate

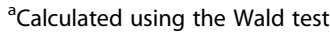

carcinomas including CRC [20]. Although GLUT1 and GLUT3 have many similarities, including expression pathways, the effects of GLUT3 on outcomes in several cancer varieties are not as well understood as GLUT1. This is the first report to study the effects of $S L C 2 A 3$ on CRC patients' survival.

The following reasons may explain why the expression of $S L C 2 A 3$ affects CRC prognosis. GLUT3 is induced by hypoxia-inducible factor (HIF) formation in response to hypoxia in carcinomas [11]. HIF-1 promotes tumor metastasis into distant and more oxygenated tissue through the transcriptional activation of oncogenic growth factors such as transforming growth factor beta3, epidermal growth factor, and others [21]. Solid tumors with high hypoxia levels are more malignant, more likely to metastasize, and have a worse prognosis [11]. GLUT3 is also induced by Akt involved in the Warburg effect. In cancer cells, Akt increases expression of GLUT1 and GLUT3 by causing degradation of p53.It may reflect the activity of hypoxia independent oncogenic pathways [11, 19].

SLC2A1 expression was associated with poor DFS but not with OS. This corresponds well with a study which reported that GLUT-1 expression is associated with poor DFS but not with OS in rectal cancer patients [9]. To further evaluate the association between the expression of $S L C 2 A 1$ and rectal cancer, we performed a subgroup analysis for rectal cancer patients using a univariate Cox regression analysis. Although we were unable to confirm our findings in the validation set due to a lack of primary site information, in the discovery set, $S L C 2 A 1$ expression in rectal cancer patients $(n=72)$ was significantly associated with DFS (HR 1.57, 95\% CI: 1.04-2.38; $P=0.03$ ). We also found no association between $S L C 2 A 1$ expression and OS (HR 1.54, 95\% CI: $0.96-2.46 ; P=0.07)$ in rectal cancer patients. Our findings were consistent with the findings of the previous study.

In addition to $S L C 2 A$ family genes, the following 12 genes are known to be involved in glucose metabolism:
MTOR, RICTOR, HIF1A, MYC, PDK1, PDK2, PDK3, PDK4, PIK3R1, PKM, POU2F-1, and RPTOR [12]. We conducted an analysis to evaluate if the expression of the genes was associated with survival outcomes of CRC. We found no association between the other 12 genes' mRNA expression and survival outcomes of CRC in both discovery and validation sets (Additional file 1: Table S1). Based on these findings, we concluded that among the glucose metabolism regulating genes, only the $S L C 2 A 3$ gene is significantly associated with the survival outcomes of CRC.

There are several genes known to be associated with the prognosis of CRC: BRAF, KRAS, HIF, TP53 and thymidylates synthase (TYMS) [22, 23]. The BRAF mutation generates an abnormality in the MEK/ERK signaling pathway in CRC [24] and has been reported to be associated with poor prognosis by many CRC studies. However, reports vary on its association with survival [25]. Mutation of KRAS, a proto-oncogene, activates $R A S$ signaling pathways, but its association with CRC survival is not clear $[24,26]$. In the current study, we analyzed the association of $B R A F$ and $K R A S$ expression level with survival, and found neither to be associated with OS or DFS in the discovery set (Additional file 1: Table S2, Table S3 and Additional file 2: Figure S1). Likewise, in the recent study conducted by authors' group, the mutational status of BRAF or KRAS was not associated the prognosis of CRC [27]. For HIF, TP53 and TYMS, there are studies which reported the expression of the genes are not related to the prognosis of OS of CRC [28-30]. In this study, we also found that HIF, TP53 and TYMS were not associated with OS or DFS (Additional file 1: Table S4). The discrepancies among the studies regarding the prognostic value of these genes may result from differences in patient cohorts, available co-variates, or statistical methods.

The short observation period of the discovery set patients (31.4 months median follow-up period) is a limitation of our study. Secondly, the relationship between the expression of $S L C 2 A$ mRNA and GLUT protein in CRC 
(a)

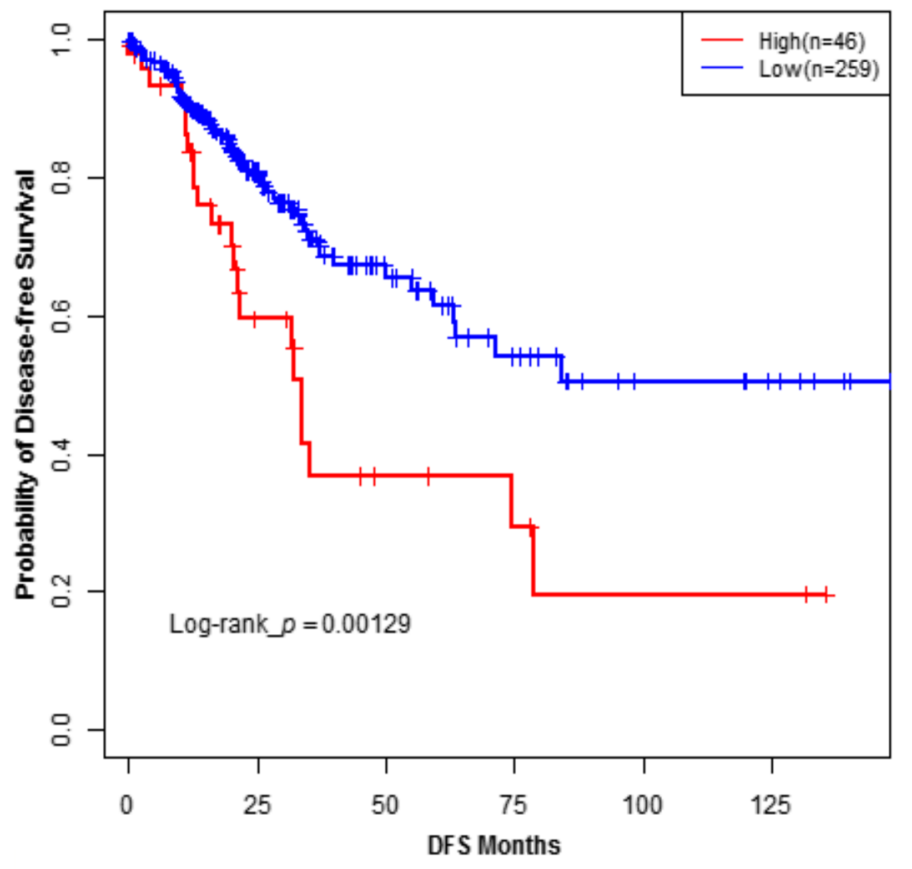

(b)

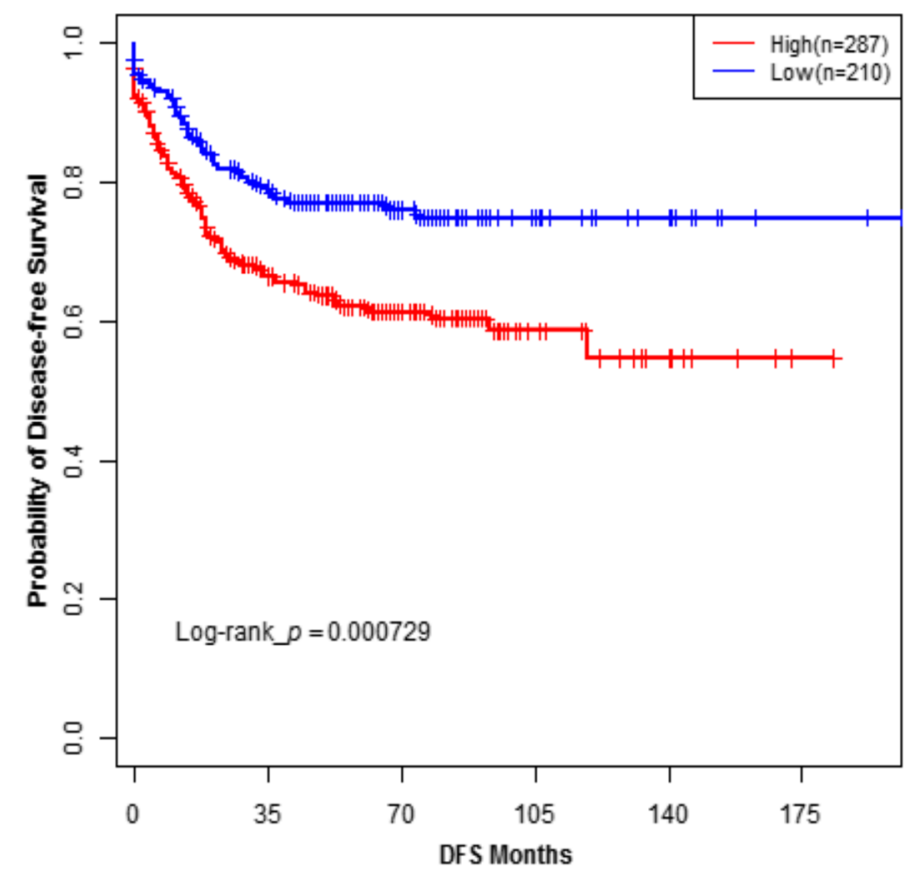

Fig. 3 Kaplan-Meier survival analysis of colorectal cancer patients stratified by SLC2A1 expression levels in Discovery set and Validation set. Disease-free survival curve of patients in Discovery set (a) and Validation Set (b) with high versus low SLC2A1 expression levels. P-values for significance of difference between high and low expression were calculated using the log-rank test 
has not been confirmed. Although one study reported a close correlation between the expression of SLC2A1/ $S L C 2 A 3$ mRNA and that of GLUT1/GLUT3 proteins in thyroid carcinoma [31], further studies are needed to investigate whether the expression of $S L C 2 A$ mRNA correlates with expression of GLUT protein mRNA in CRC. Another limitation of our results is that mRNA gene expression value is not a readily available parameter, especially in clinical settings, due to the high cost of storage and processing of fresh tissue. Its application may become wider in the near future when cost is reduced and stable mRNA expression can be obtained through formalin-fixed paraffin-embedded tissue samples.

\section{Conclusions}

In conclusion, upregulation of the $S L C 2 A 3$ gene is associated with decreased OS and DFS in CRC patients. $S L C 2 A 3$ gene expression analysis may be useful for predicting prognosis and survival of CRC patients.

\section{Additional files}

\begin{abstract}
Additional file 1: Table S1. Univariate cox regression analysis of glycolysis related genes for OS and DFS Table S2. Univariate and multivariate Cox regression analyses of BRAF mutation and the clinicopathological factors in the discovery set (TCGA COADREAD cohort) Table S3. Univariate and multivariate Cox regression analyses of KRAS mutation and the clinicopathological factors in the discovery set (TCGA COADREAD cohort) Table S4. Univariate Cox regression analysis of HIF, TP53, TYMS genes for OS and DFS in the discovery set (TCGA COADREAD cohort) (DOCX $25 \mathrm{~kb}$ )

Additional file 2: Figure S1. Kaplan-Meier Survival analysis for overall survival and disease-free survival in colorectal cancer patients according to mutational status in the Discovery set. Overall survival (a) and disease-free survival (b) according to BRAF mutation status. Overall survival (c) and disease-free survival (d) according to KRAS mutation status. Figure S2. R code used for statistical analysis. The datasets analyzed during the study are available in the following repositories: TCGA COADREAD : cBioportal for cancer genomics (http://www.cbioportal.org.). GSE39582: Gene Expression Omnibus (GEO)(https:// www.ncbi.nlm.nih.gov/geo/.) (PDF $122 \mathrm{~kb}$ )
\end{abstract}

\begin{abstract}
Abbreviations
AJCC: American Joint Committee on Cancer; BRAF: v-Raf murine sarcoma viral oncogene homolog B1 genes; Cl: Confidence interval; CRC: Colorectal cancer; DFS: Disease-free survival; GLUT: Glucose transporter; HR: Hazard ratio; IHC: immunohistochemical; KRAS: v-Ki-ras2 Kirsten rat sarcoma viral oncogene homolog; OS: Overall survival; SLC2A: Solute carrier 2A; TCGA: the cancer genome atlas
\end{abstract}

\section{Acknowledgements}

Not applicable.

\section{Funding}

This research was supported by a grant from the National Research Foundation of Korea (NRF) funded by the Korean government (Ministry of Science, ICT \& Future Planning, NRF-2016R1E1A1A01942072, http://www.nrf. re.kr/index) to YJC and the Korea Health Technology R\&D Project through the Korea Health Industry Development Institute (KHIDI), funded by the Ministry of Health \& Welfare, Republic of Korea (grant number: HI17C0048) to KK. The funding bodies had no involvement in the design of the study, collection, analysis, and interpretation of data and in writing the manuscript.

Availability of data and materials

Not applicable.

\section{Authors' contributions}

EK, SJ, WSP, JHL, RS, SCH, EKC, KK and YJC designed this research. EK, SJ, KK and YJC extracted data. SJ and KK managed data and made statistical analyses. EK, SJ wrote the first draft of the manuscript. EK, SJ and YJC revised the manuscript. All authors contributed to the interpretation of findings and wrote the final version of the manuscript. The final version was read and approved by all authors.

\section{Ethics approval and consent to participate}

Not applicable.

\section{Consent for publication}

Not applicable.

\section{Competing interests}

All authors declare that they have no competing interests.

\section{Publisher's Note}

Springer Nature remains neutral with regard to jurisdictional claims in published maps and institutional affiliations.

\section{Author details}

${ }^{1}$ Department of Surgery, National Medical Center, Seoul, Republic of Korea. ${ }^{2}$ Division of Clinical Bioinformatics, Biomedical Research Institute, Seoul National University Hospital, Seoul, Republic of Korea. ${ }^{3}$ Department of Surgery, Graduate College of Medicine, Kyung Hee University, Seoul, Republic of Korea. ${ }^{4}$ Department of Surgery, Gachon University Gil Medical Center, Incheon, Republic of Korea. ${ }^{5}$ Department of Surgery, Seoul Metropolitan Government - Seoul National University Boramae Medical Center, 20 Boramae-ro 5-gil, Dongjak-gu, Seoul 156-70, Republic of Korea. ${ }^{6}$ Department of Surgery, Seoul National University Hospital Healthcare System, Gangnam Center, Seoul, Republic of Korea. ${ }^{7}$ Department of Statistics, Korea University, Seoul, Republic of Korea.

Received: 19 September 2018 Accepted: 14 March 2019

Published online: 03 April 2019

\section{References}

1. Ferlay J, Soerjomataram I, Ervik M, et al. GLOBOCAN 2012 v1.0, Cancer incidence and Mortaligy worldwide: IARC Cancer Base no. 11. Lyon, France: International Agency for Research on. Cancer. 2013.

2. Arnold M, Sierra MS, Laversanne M, Soerjomataram I, Jemal A, Bray F. Globa patterns and trends in colorectal cancer incidence and mortality. Gut. 2017; 66(4):683-91.

3. Tariq K, Chias K. Colorectal cancer carcinogenesis: a review of mechanisms. Cancer Biol Med. 2016;13(1):120-35.

4. Lech $\mathrm{G}$, Słotwiński R, Słodkowski M, et al. Colorectal cancer tumour markers and biomarkers: recent therapeutic advances. World J Gastroenterol 2016 7; 22(5):1745-1755.

5. Luo HY, Xu RH. Predictive and prognostic biomarkers with therapeutic targets in advanced colorectal cancer. World J Gastroenterol. 2014;20(14): 3858-74.

6. Amann T, Maegdefrau U, Hartmann A, et al. GLUT1 expression is increased in hepatocellular carcinoma and promotes tumorigenesis. Am J Pathol. 2009;174(4):1544-52.

7. Younes M, Brown RW, Stephenson M, Gondo M, Cagle PT. Overexpression of Glut1 and Glut3 in stage I nonsmall cell lung carcinoma is associated with poor survival. Cancer. 1997;80(6):1046-51.

8. Chai YJ, Yi JW, Oh SW, et al. Upregulation of SLC2 (GLUT) family genes is related to poor survival outcomes in papillary thyroid carcinoma: analysis of data from the Cancer genome atlas. Surgery. 2017;161(1):188-94.

9. Yang J, Wen J, Tian T, et al. GLUT-1 overexpression as an unfavorable prognostic biomarker in patients with colorectal cancer. Oncotarget. 2017; 8(7):11788-96

10. Therneau, R. A package for survival analysis in S. R package version 2.41-3. 2017 Available at: http://CRAN.R-project.org/package=survival.

11. Barron CC, Bilan PJ, Tsakiridis T, Tsiani E. Facilitative glucose transporters: implications for cancer detection, prognosis and treatment. Metabolism. 2016;65(2):124-39.

12. Cairns RA, Harris IS, Mak TW. Regulation of cancer cell metabolism. Nat Rev Cancer. 2011;11(2):85-95. 
13. Zhao Q, Shi X, Xie Y, Huang J, Shia B, Ma S. Combining multidimensional genomic measurements for predicting cancer prognosis: observations from TCGA. Brief Bioinform. 2015;16(2):291-303.

14. Aquina CT, Mohile SG, Tejani MA, et al. The impact of age on complications, survival, and cause of death following colon cancer surgery. $\mathrm{Br} J$ Cancer. 2017;116(3):389-97.

15. Majano $S B$, Girolamo CD, Rachet $B$, et al. Surgical treatment and survival from colorectal cancer in Denmark, England, Norway, and Sweden: a population-based study. Lancet Oncol. 2018. https://doi.org/10.1016/S14702045(18)30646-6.

16. Peyravian N, Larki P, Gharib E, et al. The application of gene expression profiling in predictions of occult lymph node metastasis in colorectal Cancer patients. Biomedicines. 2018;6(1):27.

17. Ayala FR, Rocha RM, Carvalho KC, et al. GLUT1 and GLUT3 as potential prognostic markers for Oral squamous cell carcinoma. Molecules. 2010;15(4): 2374-87.

18. Baer S, Casaubon L, Schwartz MR, Marcogliese A, Younes M. Glut3 expression in biopsy specimens of laryngeal carcinoma is associated with poor survival. Laryngoscope. 2002;112(2):393-6.

19. Gjedde, A. Glucose metabolism. In Principles and Practice of Nuclear Medicine, 2nd ed.; Wagner. H., Ed.; W B Saunders: Philadelphia, PA, USA, 1995:56-62.

20. Simpson IA, Dwyer D, Malide D, Moley KH, Travis A, Vannucci SJ. The facilitative glucose transporter GLUT3: 20 years of distinction. Am J Physiol Endocrinol Metab. 2008;295(2):E242-53.

21. Masoud GN, Li W. HIF-1a pathway: role, regulation and intervention for cancer therapy. Acta Pharm Sin B. 2015;5(5):378-89.

22. Lech G, Słotwiński R, Słodkowski M, KRASnodębski IW. Colorectal cancer tumour markers and biomarkers: recent therapeutic advances. World J Gastroenterol. 2016;22(5):1745-55.

23. Aghagolzadeh P, Radpour R. New trends in molecular and cellular biomarker discovery for colorectal cancer. World J Gastroenterol. 2016; 22(25):5678-93.

24. Ahn TS, Jeong D, Son MW, et al. The BRAF mutation is associated with the prognosis in colorectal Cancer. J Cancer Res Clin Oncol. 2014;140(11):1863-71.

25. Sanz-Garcia E, Argiles G, Elez E, Tabernero J. BRAF mutant colorectal cancer: prognosis, treatment, and new perspectives. Ann Oncol. 2017;28(11):2648-57.

26. Phipps Al, Buchanan DD, Makar KW, et al. KRAS-mutation status in relation to colorectal cancer survival: the joint impact of correlated tumour markers. Br J Cancer. 2013;108(8):1757-64.

27. Lee JY, Ahn J, Park WS, et al. Colorectal cancer prognosis is not associated with BRAF and KRAS mutation-a STROBE compliant study. J Clin Med. 2019; $8(1): 111$.

28. Goto T, Shinmura K, Yokomizo K, et al. Expression levels of thymidylate synthase, dihydropyrimidine dehydrogenase, and thymidine phophorylase in patients with colorectal cancer. Anticancer Res. 2012;32:1757-62.

29. Morikawa T, Kuchiba A, Liao X, et al. Tumor TP53 expression status, body mass index, and prognosis in colorectal cancer. Int J Cancer. 2012;131(5): 1169-78.

30. da Costa AC, Stevanato Filho PR, Aguiar Júnior S, et al. Prognostic value of factors associated with hypoxia and lipid metabolism in patients with colorectal cancer. Applied Cancer Research. 2017;37:44.

31. Jóźwiak P, Krześlak A, Pomorski L, Lipińska A. Expression of hypoxia-related glucose transporters GLUT1 and GLUT3 in benign, malignant and nonneoplastic thyroid lesions. Mol Med Rep. 2012;6:601-6.

Ready to submit your research? Choose BMC and benefit from:

- fast, convenient online submission

- thorough peer review by experienced researchers in your field

- rapid publication on acceptance

- support for research data, including large and complex data types

- gold Open Access which fosters wider collaboration and increased citations

- maximum visibility for your research: over $100 \mathrm{M}$ website views per year

At BMC, research is always in progress.

Learn more biomedcentral.com/submissions 